\title{
Self-imaging effect in multimode waveguides with longitudinal periodicity
}

Stefan F. Helfert

stefan.helfert@fernuni-hagen.de

Birgit Huneke

Jürgen Jahns
Optical Information Technology, Fern Universität in Hagen, Germany

Optical Information Technology, Fern Universität in Hagen, Germany

Optical Information Technology, Fern Universität in Hagen, Germany

This paper deals with the self-imaging effect in multimode waveguides. Analogies and differences to the free-space case are shown. The fields in multimode waveguides are studied. Particularly, the behavior of the fields is examined, when a periodic perturbation is introduced. [DOI: $10.2971 /$ jeos.2009.09031]

Keywords: self-imaging effect, Talbot-effect, Montgomery condition, multimode waveguide interference, periodic structures

\section{INTRODUCTION}

For communications and processing of signals at very high data rates of $>1 \mathrm{~Tb} / \mathrm{s}$ per individual channel, one is interested in all-optical implementations since this exceeds the bandwidth of current electronic devices [1]. In particular, specific operations like signal distribution in its various uses (as fanout, clock distribution, for filter operations, etc.) can be implemented at optical frequencies without the need for impedance matching or specific considerations to the signal bandwidth. Already at lower data rates (in the range of 10$100 \mathrm{~Gb} / \mathrm{s}$ ), optics is of interest in order to alleviate systemrelated bottlenecks in the interconnection of computing systems [2].

Optics can be implemented using free-space or waveguide propagation. Both offer different implementations (discrete or integrated, for example) and specific features that may be favorable depending on the purpose. Free-space optics uses the third dimension which is suitable for a large degree of parallelism and for realizing specific interconnection tasks like beam-splitting and coupling, for example [3]. Waveguide optics, on the other hand, allows for compact devices that also include active functionality. Recent advances in the field of nano-optics, in particular, photonic crystals, for example, may even lead to ultra-compact waveguide structures [4].

A particular waveguide-optical component is the so-called "multimode interference device" or, briefly, MMI. This is essentially a planar dielectric multimode waveguide. Due to the multimode operation and the inherent periodicity of waveguides in transverse direction, self-imaging of the wavefield occurs. This can be used to implement image transmission [5, 6] and beam-splitting [7], for example. The MMI is at the beginning of the work to be presented here. However, we go beyond the structure of a conventional MMI to investigate multimode waveguides with additional longitudinal period- icity [8]. In other words, the wavefield has to obey two different conditions (lateral and longitudinal periodicity) and we want to find out how such wavefields propagate. Two questions arise: a) how do such devices work? and b) what can they be used for? Here, we look at a) and hope to answer b) later.

For this investigation, multimode waveguides with added longitudinal periodicity are simulated. We are assuming devices where the cross-section is essentially one-dimensional so that a 2D model can be used restricted to TE-polarization. The periodic disturbances in this case consisted of simple dents. As a simulation method we used the method of lines (MoL) which is well suited to yield very good results for such structures [9]-[11]. The MoL is an eigenmode algorithm, where the eigenmodes are determined after a discretization with finite differences. This means, the field is determined at discrete positions in space. After determination of the eigenmodes, all further analysis can be done analytically. This permits to take into account the essential physical features. The ones that are important for the problem to be treated here are reflections and radiation. It should be noted, that the model used is not restricted to the paraxial case but can be used at arbitrary angles.

The paper is organized as follows: in Section 2, we briefly discuss self-imaging phenomenon since this is exploited in our device structure. In particular, we present a comparison between a free-space and waveguide-optical implementation. Furthermore, we consider the influence of given lateral and longitudinal periodicity on the wavefield. Section 3 is the main part of the article and deals with propagation in periodically disturbed multimode waveguides. Results of simulations are presented and discussed. Section 4 summarizes the paper. 


\section{BRIEF REVIEW OF OPTICAL SELF-IMAGING}

Self-imaging means that a wavefield $u(x, z)$ is periodic in $z$ direction, i.e. $u(x, z)=u\left(x, z+p_{z}\right)$. (Note: we restrict the discussion to the the $2 \mathrm{D}$ case throughout the paper, for simplitity.) A sufficient condition for periodicity in z-direction is given if the wavefield is laterally periodic. This can be expressed as

$$
u(x, z)=u\left(x+p_{x}, z\right) \quad \Rightarrow \quad u(x, z)=u\left(x, z+z_{\mathrm{T}}\right)
$$

This describes the widely known case of "Talbot self-imaging" [12]. The longitudinal period $p_{z}$ is usually called the "Talbot distance" and denoted by $z_{\mathrm{T}}$ :

$$
p_{z}=z_{\mathrm{T}}=2 p_{x}^{2} / \lambda
$$

Here, $p_{x}$ is the lateral period and $\lambda$ the wavelength. We should mention that the Talbot effect is limited to paraxial wavefields. Using free-space optics, the Talbot-effect can be observed for the situation of near-field diffraction behind a grating. In waveguide optics, Talbot self-imaging occurs in multimode waveguides. In the following, we discuss both cases.

\subsection{Self-imaging in free space optics}

When a grating with period $p_{x}$ is illuminated with a plane wave of wavelength $\lambda$, the different diffraction orders form an interference pattern in the near-field. For distances behind the grating given as multiples of $z_{\mathrm{T}}$, the phases of the diffraction orders add up constructively and the resulting field is the same as in the plane of the grating. This can be described mathematically by using the following formalism with the wave vectors.

The incoming wave is given as

$$
u(z)=\exp \left(i k_{z} z\right)
$$

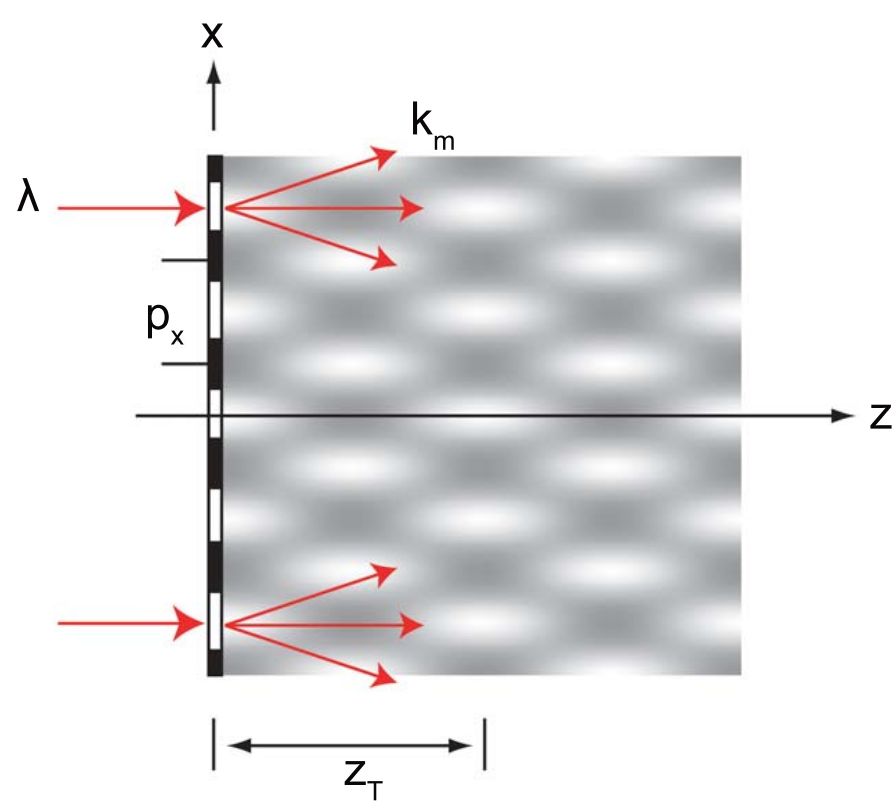

FIG. 1 Illumination of grating with a plane wave; the periodic pattern of the field repeats itself in the Talbot distance $z_{T}$.
Behind the grating, the field is

$$
u(x, z>0)=\sum_{m} a_{m} \exp \left\{i\left(k_{x}^{m} x+k_{z}^{m} z\right)\right\}
$$

Here, $a_{m}$ is the amplitude of the $m$-th diffraction order $k_{x}^{m}$ and $k_{z}^{m}$ are the components of the corresponding $k$-vector $\mathbf{k}_{m}$. It is

$$
k_{x}^{m}=m 2 \pi / p_{x}
$$

Due to the separation condition in 2D (i.e. for $k_{y}=0$ ):

$$
\left(k_{x}^{m}\right)^{2}+\left(k_{z}^{m}\right)^{2}=\left(\frac{2 \pi}{\lambda}\right)^{2}
$$

we obtain the following discrete values for $k_{z}$ (the longitudinal component of the wave vector):

$$
k_{z}^{m}=2 \pi \sqrt{\left(\frac{1}{\lambda}\right)^{2}-\left(\frac{m}{p_{x}}\right)^{2}} \quad \text { with } \quad m=0,1,2, \ldots
$$

Strictly speaking, the Talbot-effect is only valid for paraxial waves. In this case, the expression for $k_{z}^{m}$ can be approximated as:

$$
k_{z}^{m} \approx \frac{2 \pi}{\lambda}\left(1-m^{2} \frac{\lambda}{z_{\mathrm{T}}}\right)
$$

\subsection{Self-imaging in multimode waveguides}

Next, we would like to compare the situation in free space with that in multimode waveguides. We consider the symmetric waveguide structure shown in Figure 2. Due to the reflecting side walls, there is a "virtual" periodicity inside the waveguide which leads to a set of discrete eigenmodes. The field components of these eigenmodes in the core depend on $x$ according to:

$$
E, H \sim\left\{\begin{array}{l}
\cos \left(k_{x} x\right) \\
\sin \left(k_{x} x\right)
\end{array}\right.
$$

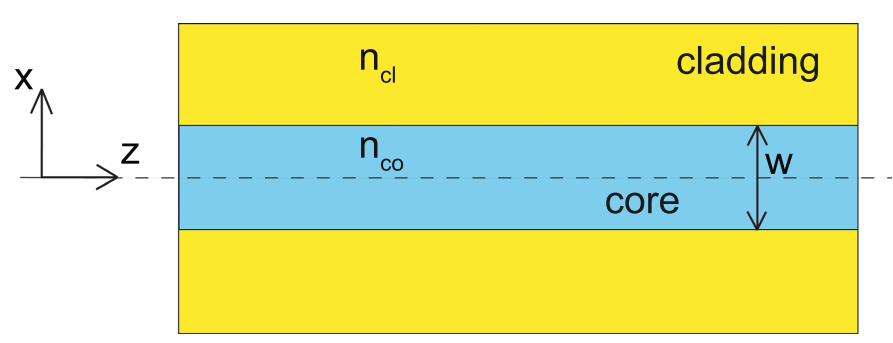

FIG. 2 Basic multimode waveguide used for the studies, parameters, $w=20 \mu \mathrm{m}$, wavelength $\lambda=1.5 \mu \mathrm{m}, n_{F}=1.52, n_{S}=1.45$.

The virtual periodicity is described by the properties of the trigonometric functions. For paraxial waves, the vertical periodicity is approximately: $p_{x} \approx 2 w$. Hence, a first estimation gives the values

$$
k_{x}^{m}=n_{\mathrm{co}} \frac{m 2 \pi}{p_{x}} \quad \text { with } \quad m=1,2, \ldots
$$

for the different modes, in analogy to the free space problem [see Eq. (5)]. However, two important points should be addressed here: a) We start with $m=1$ in multimode waveguides. The structure shown in Figure 2 supports 12 guided modes (TE-polarization) for the chosen wavelength $(\lambda=$ 
$1.5 \mu \mathrm{m})$. Hence, the maximum value of $m$ is $12 . \mathrm{b})$ In contrast to free-space optical case, the period is not the same for different modes because the fields of the different modes extend differently into the cladding. Therefore, one cannot speak of an exact transverse period for the waveguide.

Nonetheless, there is a similarity between self-imaging in a multimode waveguide and the free-space optical situation. To demonstrate this, we look at the $z$-components of the $k$-vectors for both cases. For free-space optics, the $k_{z}$-components are given by Eq. (7). For the waveguide, we determined the $k_{z^{-}}$ components by simulations with the MoL. Both sets of values are shown in Figure 3. From $k_{z}$ we determine an angle of propagation $\left(\cos \theta=k_{z} / k_{0}=\lambda k_{z} / 2 \pi\right.$ see Figure 4$)$. Obviously, there is a very good agreement between the two cases, at least, for lower order modes. The differences increase with $m$. This can be understood with the zig-zag model of wave propagation. (This model can be found in basic textbooks about optical waveguides, so we do not go into details here.) As known, the phase change at the film/substrate interface decreases with increasing angle $\theta$ (see Figure 3 ). For waves propagating strictly in $z$-direction $(\theta=0)$ we have a phase change of $\pi$ at the boundary. Therefore, the virtual periodicity of the waveguide corresponds to twice the thickness of the film layer $(=2 w$ in Figure 3) and as mentioned previously, we introduced this value for $p_{x}$ in Eq. (7). Now, as also known, the phase change decreases to zero at the limiting angle of total internal reflection. Hence, the virtual periodicity of the waveguide depends not only on its width but also on the mode number.

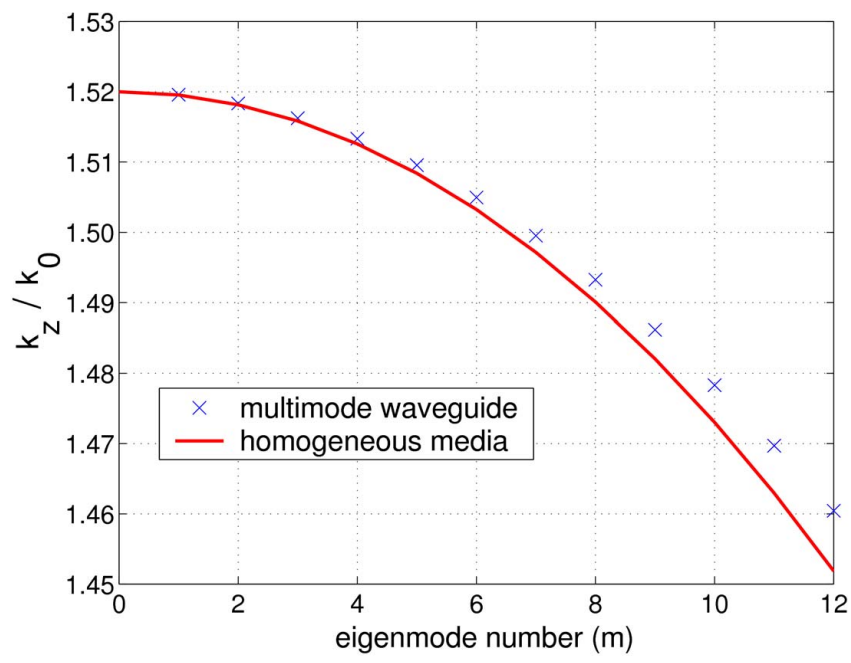

FIG. 3 Normalized longitudinal component of the wave vector, comparison of a homogeneous media ( $n=1.52$ ) with values of the multimode waveguide in Figure 2.

We have mentioned earlier, that multimode waveguides have been used as waveguide couplers in form of the so-called MMI device [7]. We will use the conventional MMI here as a reference for our later analysis. To check the validity of our numerical tools and to reconfirm the results from the literature, we started with examining the self imaging-effect in such multimode waveguides. We injected the fundamental mode of a thin monomode waveguide and computed the electric field distribution.

Figure 5 shows the determined field distribution for a symmetric input field (i.e. in the center of the multimode waveg-

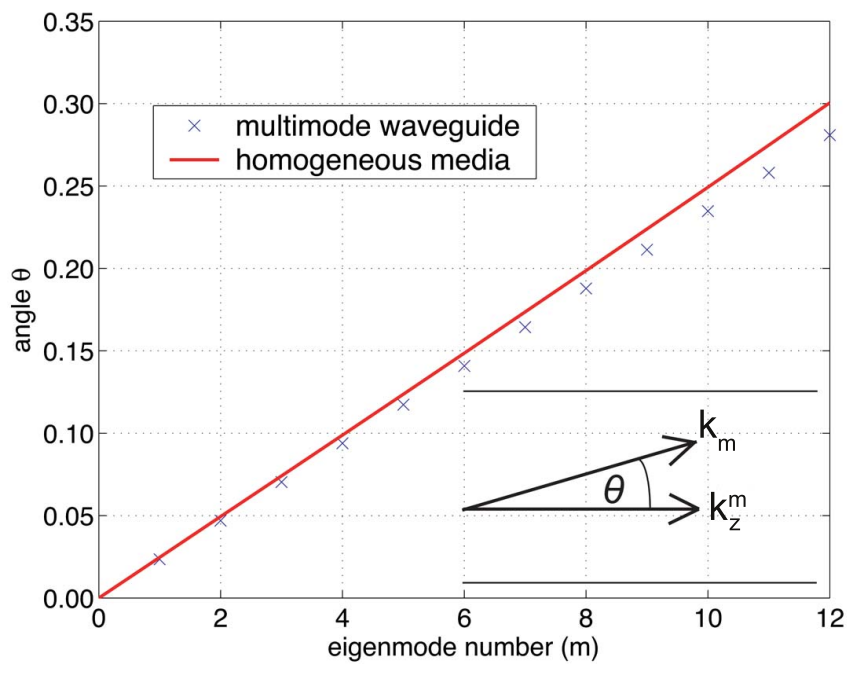

FIG. 4 Angle of propagation with respect to the optical axis, angle determined from $k_{z}$ (see Figure 3).

uide). The field for an asymmetric excitation is presented in Figure 6 . Let us start with the latter results. We see a repetition of the input field at $z_{T} \approx 3600 \mu \mathrm{m}$.

In [7] is described how the repetition length can be determined from the propagation constants of the multimode waveguide.

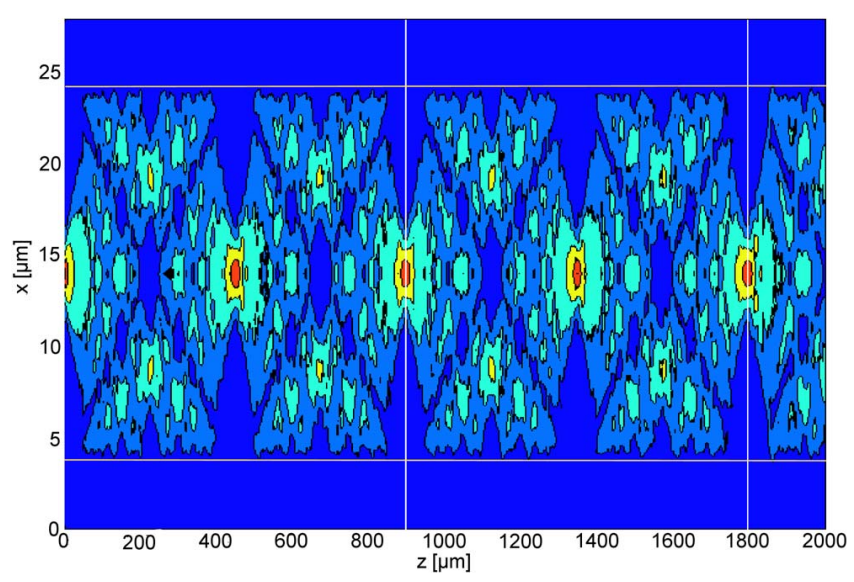

FIG. 5 Electric field distribution in a multimode waveguide, symmetric input field.

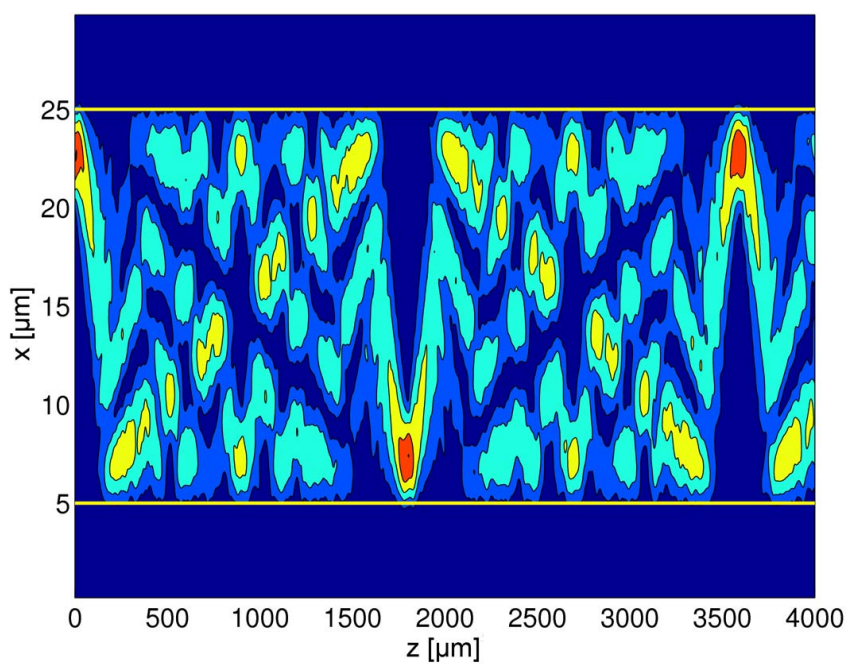

FIG. 6 Electric field distribution in a multimode waveguide, asymmetric input field. 
If we introduce the values that we determined with the MoL, we obtain $z_{T}=3589 \mu \mathrm{m}$, which is in excellent agreement with the one that we see in Figure 5.

Now, in case of a symmetric excitation the input field repeats itself also in fractals of this length. (More theoretical details can also be found in [7]). The shortest repetition length is given as $z_{T} / 8=448 \mu \mathrm{m}$. Such a length can also be observed in Figure 5.

\subsection{Montgomery self-imaging}

Talbot self-imaging describes the situation where a lateral periodicity is imposed upon a wavefield which leads to a longitudinal periodicity. A different point of view was presented by Montgomery [13]: if a longitudinal periodicity is imposed on the wavefield by a suitable structure, then the $k$-vectors of the wavefield have to obey a certain condition. This can be expressed in the following way:

$$
u(x, z)=u\left(x, z+p_{z}\right) \Rightarrow u_{0}=\sum_{m} A_{m} \exp \left\{i\left(k_{x}^{m} x+k_{z}^{m} z\right)\right\}
$$

with

$$
k_{x}^{m}=2 \pi \sqrt{\left(\frac{1}{\lambda}\right)^{2}-\left(\frac{m}{p_{z}}\right)^{2}} \quad m=1,2, \ldots
$$

The analogy of the Montgomery condition as expressed by Eq. (12) with Eq. (7) is obvious. A graphical explanation of this analogy using the concept of the Ewald sphere was given in [14]. We would like to mention here that the Montgomery condition is valid also for non-paraxial waves in contrast to the Talbot-effect.

Here, we consider the situation where both, a lateral and a longitudinal periodicity is imposed upon the wavefield. As we know from the discussion, the lateral periodicity $p_{x}$ leads to a longitudinal period of the wavefield, $z_{T}$. On the other hand, the longitudinal periodicity of the device, $p_{z}$ may be different from the Talbot length, i.e., $p_{z} \neq z_{T}$. It is of fundamental interest to study the propagation of a wavefield under such conditions. Again, the implementation may be free-space optical, this case was analyzed in [15], or it may be waveguide-optical. This is the content of this article. The general structure to be considered is a multimode-waveguide with a periodic modulation or disturbance in the longitudinal direction as shown in Figure 7. Obviously, there exists a whole variety of possibilities to implement such structures, a few of which are indicated in the figure. We would just like to mention some of them; we could vary depth and width of the dents, the period length, the shape of the perturbation, the refractive index of the perturbated part etc.

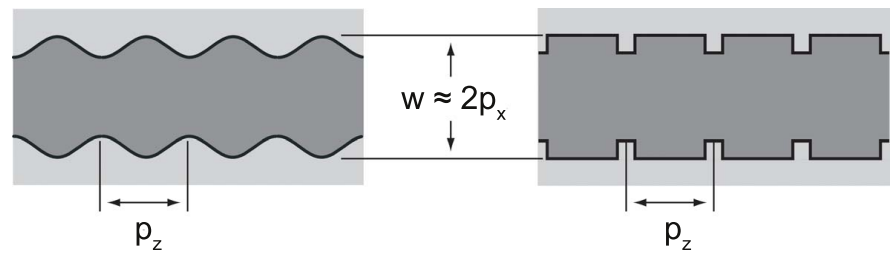

FIG. 7 Examples of waveguide implementations with longitudinal disturbance. Left: continuous, right: discrete.

\section{MULTIMODE WAVEGUIDES WITH PERIODIC PERTURBATION}

For our studies we used the multimode waveguide shown in Figure 2, in which we introduced periodic perturbations (see Figure 8). In particular, we were interested in their influence on the field distribution [8]. The monomode waveguide at the input was used to obtain a suitable input field; i.e. we injected its fundamental mode.

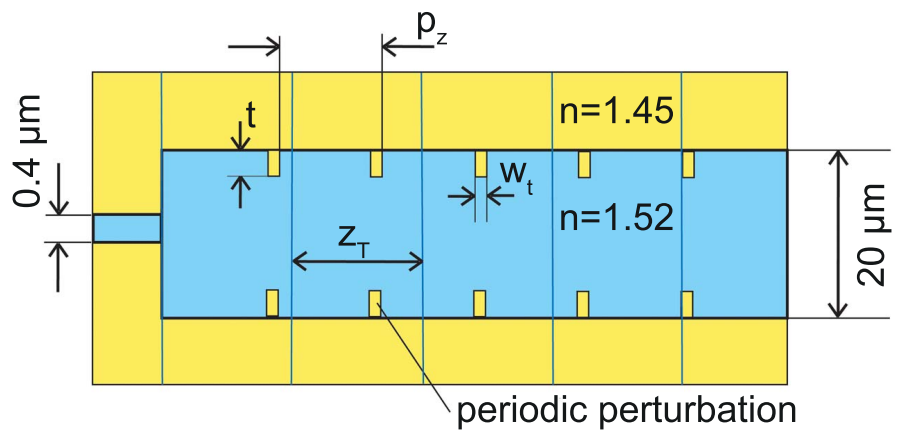

FIG. 8 Multimode waveguide with periodic perturbations.

As mentioned before, even if we assume a periodic perturbation there is a large parameter space that can be varied. Since we had to start somehow, we began with those perturbations that could be modeled most easily with our numerical algorithm. Therefore, we introduced Dirac-like rectangular teeth and examined the influence of the width $t$ and the length $z_{P}$ (see Figure 8) on the fields. For the period length $p_{z}$ we used values around $z_{T} / 8$ i.e. perturbations in the area where the first repetition of the symmetric input field takes place.

Results for $z_{P}=0.8 z_{T} / 8$ are shown in Figure 9. Here we used different values for the depth of the teeth. The number of periods (teeth) was 10 . The non-disturbed field shows the periodic repetition of the fields as we had seen before. Now, for $t=8 \mu \mathrm{m}$ (field 4 ) we have nearly the same field distribution, with a repetition of the focus in the distance $z_{T} / 8$. The focus position, however, is shifted to the right. For smaller teeth $(t=4 \mu \mathrm{m}$ (field 2), $t=6 \mu \mathrm{m}$ (field 3) we recognize a clear disturbance of the field distribution; instead of one focus we obtain two foci in a short distance from each other. Similar qualitative results were also found for other values of $z_{P}$. These results show that such perturbations permit the modification of the field distribution.

Besides the qualitative results, we would also like to have quantitative descriptions of the influence of the perturbations. Since the self-imaging effect can be described with the interference of modes (also in our numerical model), we examined the influence on these eigenmodes.

We computed the phases of the eigenmodes $1000 \mu \mathrm{m}$ behind the last perturbation. It should be mentioned here that the absolute values of the individual phases are not important, (we could e.g. always normalize the eigenmodes at the input in such a way that their phase is a multiple of $2 \pi$ at a certain position). Rather, we are interested in the change of these phases as function of the depth $t$. Due to the symmetric input field, only the even modes were excited in this problem. We gave the fun- 

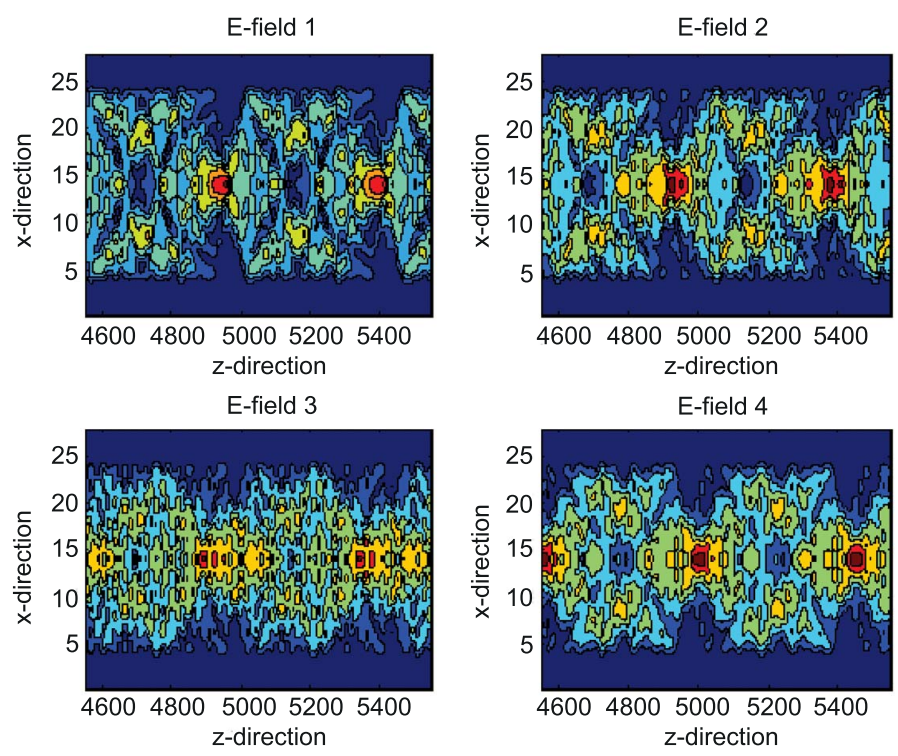

FIG. 9 Electric field distribution in the multimode waveguide Figure 2, parameters $w_{t}=1 \mu \mathrm{m}$ various depths $\Delta x$ were introduced: $0 \mu \mathrm{m}$, (field 1), $4 \mu \mathrm{m}$ (field 2), $6 \mu \mathrm{m}$ (field 3 ) and $8 \mu \mathrm{m}$ (field 4).

damental mode the number "1", in accordance with Figures 3 and 4 . The determined phases of the excited (guided) eigenmodes are shown in Figure 10 as histograms. We recognize e.g. a monotonic change (with $t$ ) of the lower modes, whereas e.g. the phase of the $11^{\text {th }}$ is relatively constant. Since the lower order modes are the main responsible ones for the behavior of the device, we took a closer look at them in Figure 11. As mentioned before, we are not interested in the absolute values of the phases (because an arbitrary value can be added), but merely in the change. Therefore, we chose the value for $t=4 \mu \mathrm{m}$ as reference and show the difference to that corresponding value here. We can clearly see that the variation of the phase for the first and fifth mode is greater than that for the third. Therefore, a specific influencing of the various modes appears possible.
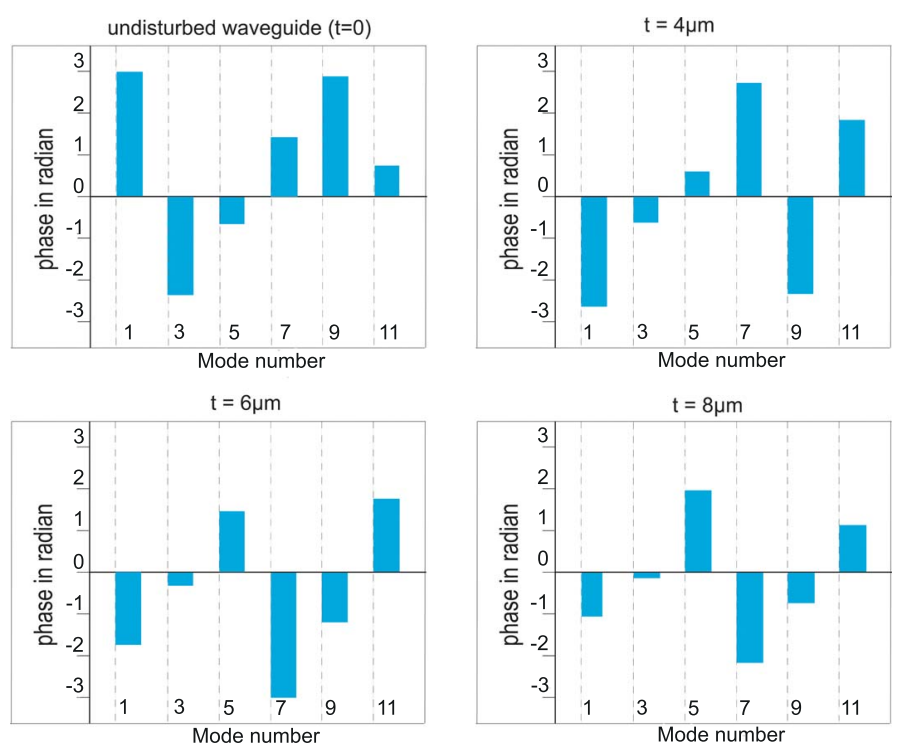

FIG. 10 Phases of the even eigenmodes $1000 \mu \mathrm{m}$ behind the periodic perturbation, shown for various values of $t$.

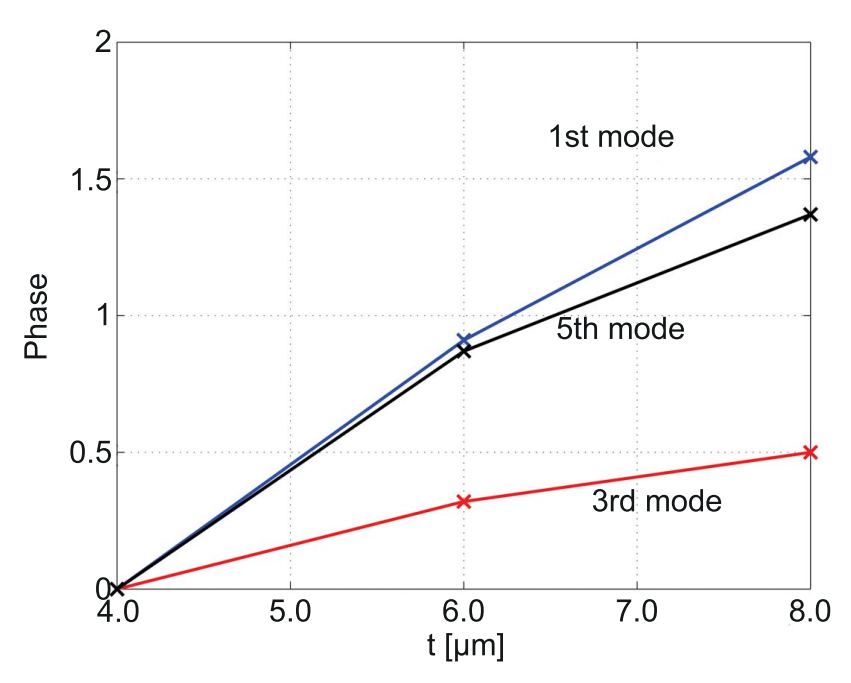

FIG. 11 Change of the phase of the lower order eigenmodes with depth $t$ of the teeth; the value for $t=4 \mu \mathrm{m}$ was taken as reference.

\section{SUMMARY}

In this work we studied the self-imaging effect in multimode waveguides. There are various aspects within in this problem that can be examined. Here, we concentrated on the behavior of the fields if additional periodic perturbations are introduced. The results show that the phases of the eigenmodes (and with them the field distribution) change differently. Next, we want to modify various other parameters (that we mentioned earlier) as well, and examine their influence on the fields. A goal is to find well defined rules on how the parameters modify e.g. the eigenmodes. This might be used for the design of circuits.

Another interesting problem arises, when we compare multimode waveguides to free space structures. As we saw, the $k_{z}$ values for these cases are similar yet different. Therefore, a question is how well the repetition of the input field (also for different injected field-distributions) really is. This should also change with increasing longitudinal distance. A measure for the agreement can be obtained e.g. by a correlation with the input field.

From the dimensions of the structures one could see that we were not dealing with the bandgap effect here, though we have a longitudinal and a (virtual) transverse periodicity. However, the self-imaging effect occurs also in such photonic crystal waveguide structures [16] with the "self-imaging length" being much longer than the period of the crystal. Besides, also in metallic structures self-imaging has been observed [17]. Particularly, these $\mathrm{PhC}-$ and metallic- waveguides are potential candidates for miniaturizing optical structures, which makes them interesting for future investigations.

\section{References}

[1] M. Sarutawari, "All-optical signal processing for terabit/second optical transmission" IEEE J. Sel. Top. Quant. 6, 1363-1374 (2000).

[2] J. W. Goodman, F. J. Leonberger, S.-Y. Kung, and R. A. Athale, 
“Optical interconnections for VLSI systems" Proc. IEEE 72, 850-866 (1984).

[3] M. Jarczynski, T. Seiler, and J. Jahns, "Integrated three-dimensional optical multilayer using free-space optics" Appl. Optics 45, 63356341 (2006).

[4] J. D. Joannopoulus, R. D. Meade, and J. N. Winn, Photonic crystals - Molding the flow of light (Princeton University Press, 1995).

[5] 0. Bryngdahl, "Image formation using self-imaging techniques" J. Opt. Soc. Am. 63, 416-419 (1973).

[6] R. Ulrich and G. Ankele, "Self-imaging in homogeneous planar optical waveguides" Appl. Phys. Lett. 27, 337-339 (1975).

[7] L. B. Soldano and E. C. M. Pennings, "Optical multi-mode interference devices based on self-imaging: principles and applications" J. Lightwave Techol. 13, 615-627 (1995).

[8] S. F. Helfert, B. Huneke, and J. Jahns, "Studies of the self-imaging effect in multimode waveguides" in ICTON Conf., 255-258 (Athens, Greece, 2008).

[9] R. Pregla and W. Pascher, "The Method of Lines" in Numerical Techniques for Microwave and Millimeter Wave Passive Structures, T. Itoh, ed., 381-446 (J. Wiley Publ., New York, USA, 1989).
[10] S. F. Helfert and R. Pregla, "The method of lines: a versatile tool for the analysis of waveguide structures" Electromagnetics 22, 615637 (2002).

[11] R. Pregla, Analysis of Electromagnetic Fields and Waves - The Method of Lines (Wiley it Sons, Chichester, UK, 2008).

[12] H. F. Talbot, "Facts relating to optical science, No. IV" Philos. Mag. 9, 401-407 (1836).

[13] W. D. Montgomery, "Self-imaging objects of infinite aperture" J. Opt. Soc. Am. 57, 772-778 (1967).

[14] A. W. Lohmann, H. Knuppertz, and J. Jahns, "Fractional Montgomery effect: a self-imaging phenomenon" J. Opt. Soc. Am. A 22, 1500-1508 (2005).

[15] J. Jahns and A. W. Lohmann, "Optical wavefields with lateral and longitudinal periodicity" Appl. Opt. 48, 3438-3445 (2009).

[16] H.-J. Kim, I. Park, O. Beom-Hoan, S.-G. Park, E.-H. Lee, and S.-G. Lee, "Self-imaging phenomena in multi-mode photonic crystal linedefect waveguides: applicazion to wavelength de-multiplexing" Opt. Express 12, 5625-5633 (2004).

[17] M. R. Dennis, N. I. Zheludev, and F. J. G. de Abajo, "The plasmon Talbot effect" Opt. Express 15, 9692-9700 (2007). 